\title{
Research Title: Factors Associated with Cessation of Exclusive Breastfeeding
}

This article was published in the following Dove Press journal:

Journal of Multidisciplinary Healthcare

\author{
Amira Yousef Ali Aldalili (D) \\ Azza Ali El.Mahalli $\mathbb{D}^{2}$ \\ 'Dental Department, Primary Health \\ Care Center, National Guards Affairs, \\ Alehsa, Saudi Arabia; ${ }^{2}$ Department of \\ Public Health, College of Public Health, \\ Imam Abdulrahman Bin Faisal University, \\ Dammam, Saudi Arabia
}

Purpose: The study aimed to determine the prevalence of exclusive breastfeeding (EBF), measure the association between EBF and sociodemographic and obstetric characteristics of lactating mothers, and determine challenges of EBF.

Methods: The study was cross-sectional and was conducted in four primary health care centres (PHCCs) at Alehsa region in Saudi Arabia (SA). Lactating mothers coming to vaccinate their babies (0-6 months) were recruited. Sample size totalled 372, where 93 were randomly selected from each centre. Sociodemographic and obstetric characteristics of participants, breastfeeding (BF) status, and challenges of EBF were collected. Basic univariate descriptive statistics were conducted to explore the sociodemographic and obstetric characteristics, BF status and challenges of BF. Bivariate analyses were done to explore the association between the dependent and independent variables. Binary logistic regression models were then executed. A 2-tailed p-value $<0.05$ was considered significant.

Results: EBF rate was nearly $60 \%$. Cessation of EBF was associated with younger age, inconvenience/fatigue due to $\mathrm{BF}$, sore breasts or nipples/too painful, perceived low milk quantity, BF skills were not effective, maternal choice, and baby-centred factors.

Conclusion and Recommendations: EBF was associated with problems/difficulties in $\mathrm{BF}$ technique. It is recommended that health care professionals like doctors, nurses, and midwives should train mothers during and after pregnancy regarding BF technique or pumping breast milk in case of BF difficulties, improve mothers' confidence about the ability to breastfeed and enhance mothers' knowledge on the normal process of lactation. Secondly, it is the role of policymakers to ensure implementation of Baby-Friendly Hospital Initiative (BFHI) guidelines in hospitals to meet the WHO's global target of infants being exclusively breastfed until six months of age.

Keywords: exclusive breastfeeding; EBF, cross-sectional study, risk factors

\section{Introduction}

$\mathrm{BF}$ is viewed as the optimal method of infant feeding that provides many benefits to both infant and mother. ${ }^{1}$ Meta-analyses indicated protection against child infections and malocclusion, increases in intelligence, and probable reductions in overweight and diabetes. ${ }^{2}$ Therefore, studying the potential factors that affect BF practices and duration is of significance. ${ }^{1}$ Extensive research has demonstrated that the long duration of BF also contributes to the good health and well-being of mothers and babies. $^{3}$ Consequently, World Health Organization (WHO) and an international body like United International Children Emergency Fund (UNICEF) implemented a number of guidelines that recommend mothers to breastfeed their babies for the first 6 months. ${ }^{4,5}$
Correspondence: Azza Ali El.Mahalli Department of Public Health, College of Public Health, Imam Abdulrahman Bin Faisal University, PO Box 1982, Dammam, 3|44I, Saudi Arabia

Tel +96654262 2475

Email amahally@iau.edu.sa
Journal of Multidisciplinary Healthcare 2021:14 239-246 
WHO defined EBF as "[when] infant is allowed to receive breast milk only without any food, drinks, even water, except drops of syrups, vitamins, minerals or medicines". ${ }^{4}$ Although the benefits of EBF have spread worldwide, $\mathrm{BF}$ is still unpopular in most parts of the world. $^{5}$

As a part of the global efforts to promote $\mathrm{BF}$, the government of SA has put a tremendous effort through adopting the Baby-Friendly Hospital Initiative (BFHI) Program to promote, protect, and support BF. Still, the outcomes are below expectation. ${ }^{6}$ Today, the rate of EBF initiation, EBF, and continued BF to 24 months are not high as expected. ${ }^{7}$ National cross-sectional studies in SA showed that BF rate declined from $93 \%$ in the first month to $89 \%$ in the second month, and then to $78 \%$ in the 6 th month. ${ }^{8}$

The main challenges impeding the success of BF were: formal work schedule; informal work; family influence due to community culture from grandmothers, cousins, and aunties; insufficient breast milk; and swollen breast or sore nipples. ${ }^{9-11}$ On the other hand, in the Gulf Cooperation Council (GCC) countries \{United Arab Emirates, Bahrain, Oman, SA, Qatar, and Kuwait $\}$, the challenges of $\mathrm{BF}$ were; inadequate training of health care staff, increased marketing of infant milk formula, lack of education and policy support, presence of only 3 out of 11 non-governmental hospitals adopting BFHI practice, short maternity leave, insufficient milk production, sickness of a lactating mother, and lactating mothers getting pregnant. $^{12}$

The literature revealed that the initiation rate of $\mathrm{BF}$ is high in SA. However, the rate declined over six months. ${ }^{1}$ To the best of the researcher's knowledge, no studies have been conducted to explore the challenges facing EBF in Alehsa region in SA. Accordingly, the study aimed to determine the prevalence of mothers who adopted EBF for 1, 2 and 6 months after delivery at Alehsa region in $\mathrm{SA}$, measure the association between EBF vs the sociodemographic and obstetric characteristics of lactating mothers and identify the challenges of EBF.

\section{Materials and Methods}

\section{Study Design, Data Source and Population}

Cross-sectional study conducted at four PHCCs in Alehsa district in SA. One PHCC was chosen from each of the four regions in Alehsa. These centres were chosen as they have the highest frequency of visits (encounters) of lactating women to vaccinate their babies (0-6 months). Similarly, the four regions of Alehsa district were represented. Study participants were lactating women visiting the centres to vaccinate their babies (0-6 months). Mothers who have single birth, 17 years and older, and gave birth after 24 gestational weeks were included. However, mothers with medical contraindications for BF, such as HIV/AIDS and babies who received intensive care monitoring and/or with malformations that interfered with their BF, were excluded.

\section{Data Collection}

Data collection sheet was designed (Appendix 1) and validated by faculty members in the family and community medicine department as well as paediatricians. The questionnaire comprised of $\mathrm{BF}$ status as the dependant variable Mothers who exclusively breast fed their babies at I, 2 and 6 months were considered as EBF and mothers who did not breastfeed their babies since birth (only formula feeding) or who used partial BF (formula $+\mathrm{BF}$ ) from the start were considered as non-EBF $\}$ and maternal sociodemographic characteristics, e.g., age, mothers' education and occupation, family income, etc., obstetric characteristics, e.g., gestational age, parity, and mode of birth, and reasons for cessation of EBF, e.g., Perceived low milk quantity, Sore breasts or nipples/Too painful, etc. as independent variables. ${ }^{9-13}$ Self-administered questionnaire was used to collect data from participants. A pilot study was conducted among fifteen women attending the dental clinic of one of the PHCCs under study. It was done to estimate the time needed to complete the data collection and check for any difficulty or misunderstanding of the questionnaire.

\section{Sample Size and Sampling Technique}

The sample size was determined by applying a one-sample proportion formula. The researcher used $55 \%$ as an average of the proportions of the previous studies. ${ }^{14}$ The precision of estimation was determined as $5 \%$. Then, the initial sample size was 423 women, and after correction for finite population, which is approximately 2880 women visiting the four centres semi-annually to vaccinate their babies, the final sample size was 372 mothers. They were distributed uniformly among the four centres; 93 women were randomly selected from each centre using systematic random sampling technique after the application of inclusion and exclusion criteria. 


\section{Statistical Analysis}

Basic univariate descriptive statistics were executed (for example; frequency counts, percentages, means, and standard deviations) to explore the socio-demographic characteristics, obstetric history, BF status, and barriers impeding mothers during their $\mathrm{BF}$ practice. Bivariate analyses were done to explore the association between the dependent and the independent variables. Dependent variables were the $\mathrm{BF}$ status, and independent variables were the sociodemographic characteristics, obstetric history, and reasons for the cessation of EBF. Then, statistically significant variables were considered as candidate variables in the binary logistic regression analysis models. $\mathrm{P}$-value $<0.05$ was considered statistically significant. Statistical analysis was completed using the SPSS 24.0 computer software package for Windows (SPSS, Inc., Chicago, IL). Ethical approval from Imam Abdulrahman Bin Faisal University's research ethical review board (IRB-PGS-2019-03-288) and written informed consent from participants were obtained. Study complies with the Declaration of Helsinki. Data obtained from the participants were kept confidential.

\section{Results}

Study findings revealed that the mean age of mothers was 30.6 years \pm 6.53 , a majority had at least a bachelor's degree $(52.7 \%)$, were not working $(75 \%)$, and had family income below 10,000 Saudi Riyal/month (58.3\%). The prevalence of mothers who adopted EBF for 1, 2 and 6 months postpartum was $16.9 \%, 21.5 \%$, and $22.0 \%$ respectively. However, those mothers that adopted non- EBF was approximately $40 \%$. The most frequent reasons for cessation of EBF were perceived as low milk quantity, baby-centred factors, maternal choice, and inconvenience/fatigue due to $\mathrm{BF}$.

Table 1 demonstrated many variables that showed a statistically significant difference between mothers adopted EBF for 1, 2 and 6 months after birth versus nonEBF regarding some factors.

Table 2 illustrated adjusted OR for EBF with respect to various independent variables. Inconvenience/fatigue due to $\mathrm{BF}$ was a risk factor associated with cessation of $\mathrm{EBF}$ at 1, 2 and 6 months after birth. Sore breasts or nipples being too painful was a risk factor associated with cessation of EBF at 2 and 6 months after birth. In addition, claims of lack of skills in BF, perceived low milk quantity, maternal choice, and baby-centred factors were risk factors associated with discontinuation of EBF at 6 months after delivery.

\section{Discussion}

The current study demonstrated that the prevalence of mothers who adopted EBF for 1, 2 and 6 months postpartum at Alehsa was $16.9 \%, 21.5 \%$, and $22.0 \%$ respectively. Findings of a review study conducted in SA and reviewed 17 cross-sectional studies revealed that EBF rates range from $0.8 \%$ to $43.9 \%{ }^{1}$ At Riyadh, SA, $51 \%$ $(\mathrm{n}=53)$ of BFHI participants experienced EBF, however $29.6 \%(n=29)$ of non-BFHI participants experienced EBF. ${ }^{6}$

Low prevalence of practising EBF could be due to lack of training of mothers during and after pregnancy regarding BF technique and or lack of mothers' awareness of the significance of BF. A systemic review was done in Iran among 32 studies, and the overall rate of EBF was 53\%. The study revealed that the relatively high prevalence of EBF was attributed to the training of mothers regarding the BF technique during and after pregnancy. Additionally, apart from most Iranian mothers being housewives, Iran's maternity leave as per its labour law is 6 months. ${ }^{15}$

In this study, mothers who cited "inconvenience/fatigue due to BF" had more chance to stop EBF. This could be inferred to the fact that $\mathrm{BF}$ was tiring or demanding for the mother in addition to the lack of a mother's time to breastfeed while caring for other children. A study done in Canada reported that the most frequent reason mentioned for a cessation of EBF was inconvenience or fatigue and it was correlated with BF by $22.6 \%$ of mothers; about $22.8 \%$ mothers ceased BF in less than 1 week, $24.5 \%$ discontinued BF from 1-6 weeks, while $18.9 \%$ stopped BF in more than 6 weeks. ${ }^{16}$

"Sore breast or nipples" was another risk factor related to the cessation of EBF. Mothers complained of painful nipples, general or unspecified BF pain, sore breasts, engorgement, breast pain, and biting. This concurs with a study conducted in two different regions in Ghana in 2019 where mothers reported that the swollen breast or sore nipples were major challenges that hindered efforts to optimum BF. ${ }^{9}$

Moreover, another study conducted at the breastfeeding centre of Western Australia also had its findings showing that nipple pain was one of the reasons for early weaning. The study recommended that effective early lactation management for prevention of nipple pain and early diagnosis and effective treatment are crucial to avoid early weaning. ${ }^{17}$ Moreover, "perceived minimal milk quantity" 
Table I Association of Cessation of EBF with Socio-Demographic Characteristics, Obstetric History, and Reasons of EBF Cessation in Alehsa, 2020

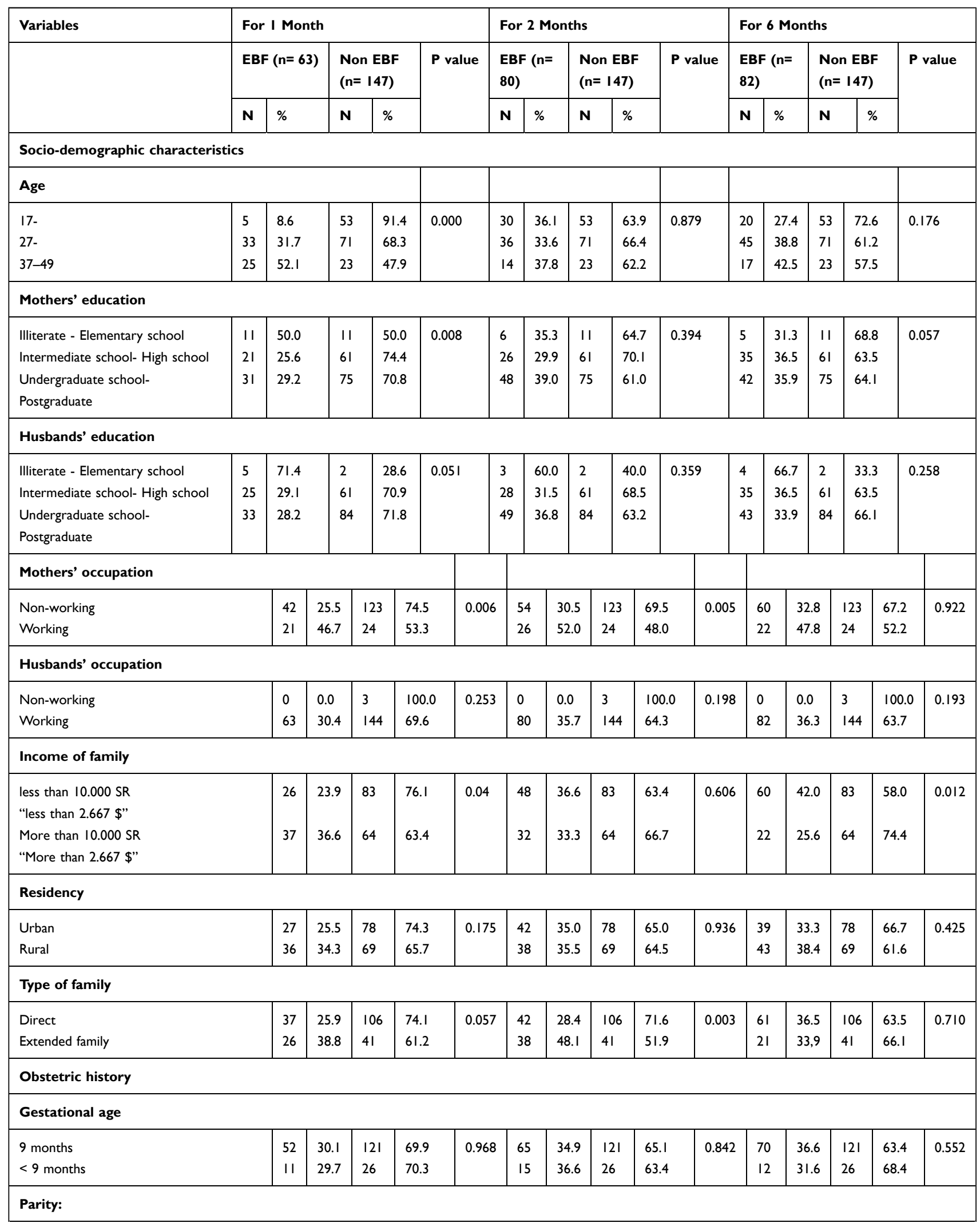

(Continued) 
Table I (Continued).

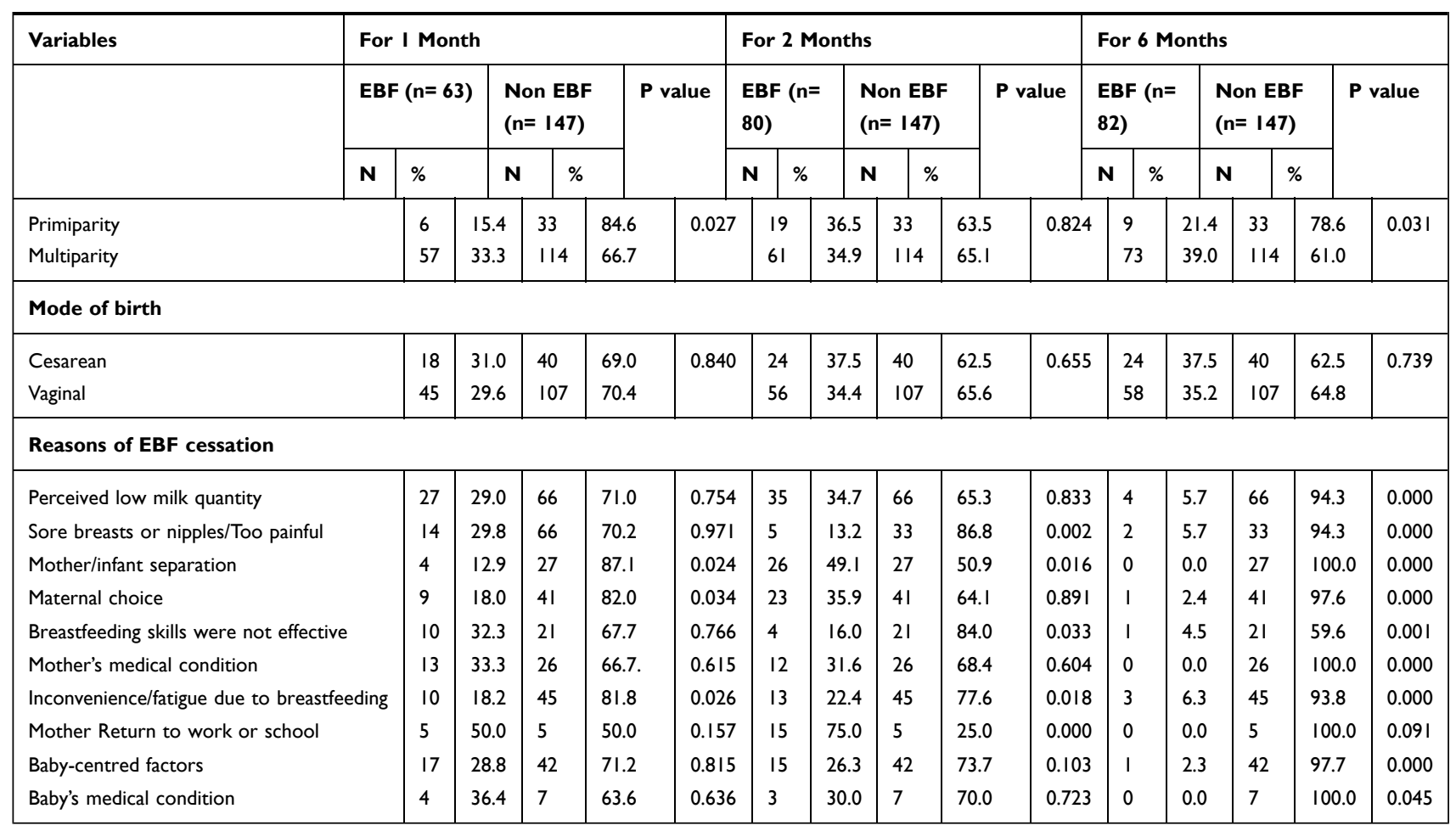

was a risk factor associated with cessation of EBF. Mothers perceived that the infant was not getting enough milk (28.5\%) and was showing signs of hunger (11.8\%). Another study presented similar result where the most popular barrier for a cessation of EBF at the early postpartum period was the perception of mothers of inadequate milk supply in the first few days; therefore, they introduced formula for their babies after pushing from hospital's staff. ${ }^{18}$ On the other hand, perceived insufficient milk comes from lack of mothers' knowledge about lactation physiology. One study addressed maternal concerns in relation to perceived insufficient milk, especially regarding the role of maternal interpretation of crying as a sign of hunger and its role as the initiator of the perceived insufficient milk cycle. The study recommended making the connection between incomplete or infrequent removal of milk from the breast and breast milk production clearer to women may play an important role in reducing the prevalence of perceived insufficient milk and its impact on EBF rates. Additionally, the study recommended manual breast milk extraction if the mother and baby were apart. ${ }^{19}$

Woman's conception of insufficient milk quantity was attributed to lack of knowledge regarding the normal process of lactation, or technical difficulties in feeding, rather than an actual inability to produce enough milk. ${ }^{20}$ Additionally, mothers who cited "BF skills were not effective" had a higher probability of stopping EBF. The findings demonstrated that $6.5 \%$ of mothers faced problems with $\mathrm{BF}$ technique, and $2.7 \%$ stopped $\mathrm{EBF}$ at 6 months because the baby was uncomfortable with the act or connotations of BF. Either others stopped EBF as their babies suffered from colic, or the mothers had trouble in BF. This also affirms results of a study done in the United States where about $28 \%$ of mothers had difficulty with $\mathrm{BF}$ and uncertainty in their ability in BF. ${ }^{21}$

Similarly, "maternal choice" is another reason to stop EBF among lactating mothers. This could be due to psychosocial distress of mothers. This is in accordance with a secondary analysis of data collected from 7,942 participants who were enrolled in a peer counselling BF support program done by Michigan State University. Mothers discontinued BF because of psychosocial distress. ${ }^{22}$ Moreover, a cross-sectional survey of women who discharged from five hospitals in Ontario, Canada revealed that mothers' intention to breastfeed for a short duration ( $<4$ months) was a key risk factor for early cessation of $\mathrm{BF}^{23}$

The final risk factor to stop EBF is referred to as "baby's centred factor". Mothers demonstrated problems 
Table 2 Factors Associated with Cessation of Exclusive Breastfeeding After I, 2 and 6 Months of Birth in Alehsa, 2020

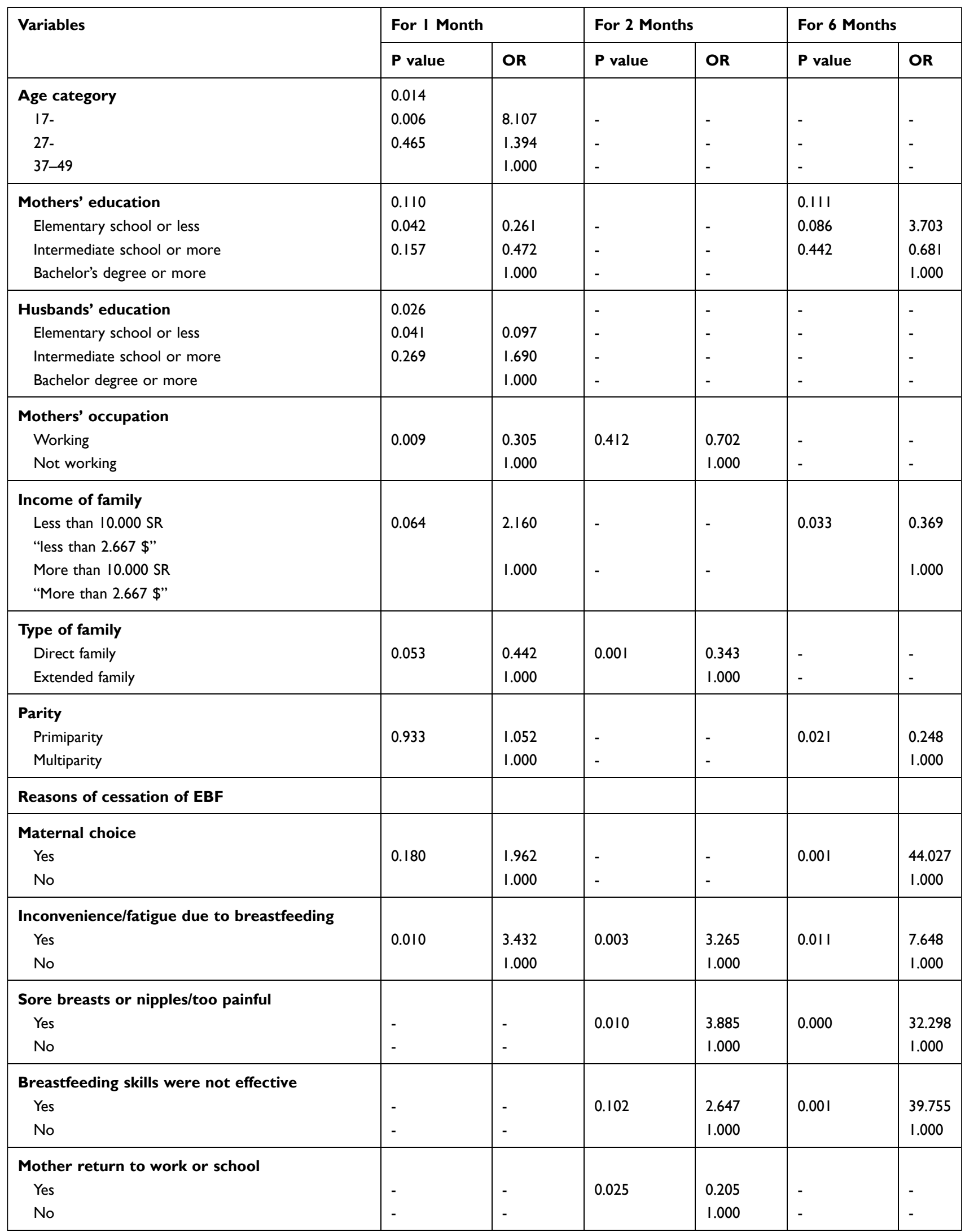

(Continued) 
Table 2 (Continued).

\begin{tabular}{|c|c|c|c|c|c|c|}
\hline \multirow[t]{2}{*}{ Variables } & \multicolumn{2}{|c|}{ For I Month } & \multicolumn{2}{|c|}{ For 2 Months } & \multicolumn{2}{|c|}{ For 6 Months } \\
\hline & $P$ value & OR & $P$ value & OR & $P$ value & OR \\
\hline \multicolumn{7}{|c|}{ Perceived low milk quantity } \\
\hline Yes & - & - & - & - & 0.000 & 12.219 \\
\hline No & - & - & - & - & & 1.000 \\
\hline \multicolumn{7}{|c|}{ Baby's-centred factors } \\
\hline Yes & - & - & - & - & 0.002 & 35.531 \\
\hline No & - & - & - & - & & 1.000 \\
\hline
\end{tabular}

with latching $(3 \%)$, infant refusal of $\mathrm{BF}(9.9 \%)$, preterm baby $(1.3 \%)$, and infant's colic $(0.3 \%)$. This agrees with a systematic review study that confirmed that infants might also reject breast and prefer the bottle because either they are having a problem with BF or they may be experiencing nipple confusion. ${ }^{24}$

Comparing our study findings with other studies in SA, a review study showed the most common reason for $\mathrm{BF}$ cessation was insufficient breast milk, sickness, new pregnancy, and BF problems. ${ }^{1}$

Limitations of this study are recall bias, inability to generalize the study results across SA and study participants are only Saudi nationals.

\section{Conclusion and Recommendations}

It is recommended that health care professionals like doctors, nurses, and midwives should train mothers during and after pregnancy regarding BF technique or pumping breast milk in case of BF difficulties, improve mothers' confidence about the ability to breastfeed and enhance mothers' knowledge on the normal process of lactation. Secondly, it is the role of policymakers to ensure implementation of BFHI guidelines in hospitals to meet the WHO's global target of infants being exclusively breastfed until 6 months of age. Thirdly, policymakers have a fundamental role in directing resources in favor of public health. Thus, it is their role to mobilize health professionals and mothers regarding the importance of EBF for society.

\section{Disclosure}

The authors report no conflicts of interest in this work.

\section{References}

1. Juaid D, Binns CW, Giglia RC. Breastfeeding in Saudi Arabia: a review. Int Breastfeeding J. 2014;9(1):1. doi:10.1186/1746-4358-9-1
2. Victora CG, Bahl R, Barros AJD, et al. breastfeeding in the 21 st century. Lancet. 2016;387(10017):475-490. doi:10.1016/S01406736(15)01024-7

3. Bhandari N, Chowdhury R. Infant and young child feeding. Proc Indian Natl Sci Acad. 2016;82(5):1507-1517. doi:10.16943/ptinsa/ 2016/48883

4. WHO. WHO | breastfeeding. Available from: http://www.who.int/ topics/breastfeeding/en/. Accessed November 26, 2020.

5. UNICEF. Infant and young child feeding - UNICEF DATA. Unicef. 2018.

6. Mosher C, Sarkar A, Hashem AA, et al. Self-reported breast feeding practices and the Baby Friendly Hospital Initiative in Riyadh, Saudi Arabia: prospective cohort study. BMJ Open. 2016;6(12):12. doi:10.1136/bmjopen-2016-012890

7. Alhabas M Breastfeeding among working mothers in Saudi Arabia. Theses Diss. 2016. https://scholarcommons.sc.edu/etd/3614.

8. Al-Jassir M, Moizuddin SK, Al-Bashir B. A review of some statistics on breastfeeding in Saudi Arabia. Nutr Health. 2003;17(2):123-130. doi: $10.1177 / 026010600301700203$

9. Tampah-Naah AM, Kumi-Kyereme A, Amo-Adjei J, Madiba S. Maternal challenges of exclusive breastfeeding and complementary feeding in Ghana. PLoS One. 2019;14(5):5. doi:10.1371/journal.pone.0215285

10. Bueno-Gutierrez D, Chantry CJ Using the socio-ecological framework to determine breastfeeding obstacles in a low-income population in Tijuana, Mexico: healthcare services. UC Davis. Available from: https://ucdavis.pure.elsevier.com/en/publications/using-thesocio-ecological-framework-to-determine-breastfeeding-o. 2015. Accessed November 26, 2020.

11. Muchacha M, Mthetwa E Beyond the bio-medical orthodoxies: socio-economic and attitudinal impediments to exclusive breast-feeding in rural Zimbabwe and possible roles of social workers and interventions to promote its uptake. Available form: https://cata log.ihsn.org/citations/83469. Accessed November 26, 2020.

12. Al-Nuaimi N, Katende G, Arulappan J. Breastfeeding trends and determinants: implications and recommendations for gulf cooperation council countries. Sultan Qaboos Univ Med J. 2017;e155-e161. doi:10.18295/squmj.2016.17.02.004

13. Chen WF, Sheng Chang PC, et al. Factors associated with cessation of exclusive breastfeeding at 1 and 2 months postpartum in Taiwan. Int Breastfeed J. 2019;14:1.

14. Tsegaye M, Ajema D, Shiferaw S, Yirgu R. Level of exclusive breastfeeding practice in remote and pastoralist community, Aysaita woreda, Afar, Ethiopia. Int Breastfeed J. 2019;14(1):1. doi:10.1186/ s13006-019-0200-6

15. Behzadifar M, Saki M, Behzadifar M, et al. Prevalence of exclusive breastfeeding practice in the first six months of life and its determinants in Iran: a systematic review and meta-analysis. BMC Pediatr. 2019;19(1):1. doi:10.1186/s12887-019-1776-0

16. Brown MC, Dodds PL, Legge MA, et al. Factors influencing the reasons why mothers stop breastfeeding. Can J Public Heal. 2014;105(3):e179-e185. doi:10.17269/cjph.105.4244 
17. Kent JC, Ashton E, Hardwick CM, et al. Nipple pain in breastfeeding mothers: incidence, causes and treatments. Int J Environ Res Public Health. 2015;12(10):12247-12263. doi:10.3390/ijerph121012247

18. Teich AS, Barnett J, Bonuck K. Women's perceptions of breastfeeding barriers in early postpartum period: a qualitative analysis nested in two randomized controlled trials. Breastfeed Med. 2013;9(1):9-15. doi:10.1089/bfm.2013.0063

19. MSacco LM, Caulfield LE, Gittelsohn J, Martínez H. The Conceptualization of Perceived Insufficient Milk Among Mexican Mothers. J Human Lactation. 2006;22(3):277-286. doi:10.1177/ 0890334406287817

20. Sun K, Chen M, Yin Y, Wu L, Gao L. Why Chinese mothers stop breastfeeding: mothers' self-reported reasons for stopping during the first six months. J Child Heal Care. 2017;21(3):353-363. doi:10.1177/1367493517719160
21. Wagner EA, Chantry CJ, Dewey KG, Nommsen-Rivers LA. Breastfeeding concerns at 3 and 7 days postpartum and feeding status at 2 months. $J$ Pediatr. 2013;132(4):865-875. doi:10.1542/ peds.2013-0724

22. Rozga MR, Kerver JM, Olson BH. Self-reported reasons for breastfeeding cessation among low-income women enrolled in a peer counseling breastfeeding support program. J Hum Lact. 2015;31 (1):129-137. doi:10.1177/0890334414548070

23. Sheehan D, Krueger P, Watt S, Sword W, Bridle B. The Ontario mother and infant survey: breastfeeding outcomes. J Hum Lact. 2001;17(3):211-219. doi:10.1177/089033440101700304

24. Zimmerman E, Thompson K. Clarifying nipple confusion. J Perinatol. 2015;35(11):895-899. doi:10.1038/jp.2015.83

\section{Publish your work in this journal}

The Journal of Multidisciplinary Healthcare is an international, peerreviewed open-access journal that aims to represent and publish research in healthcare areas delivered by practitioners of different disciplines. This includes studies and reviews conducted by multidisciplinary teams as well as research which evaluates the results or conduct of such teams or healthcare processes in general. The journal covers a very wide range of areas and welcomes submissions from practitioners at all levels, from all over the world. The manuscript management system is completely online and includes a very quick and fair peer-review system. Visit http://www.dovepress.com/testimonials. php to read real quotes from published authors. 